\title{
A TOPOLOGICAL PROOF OF THE EQUIVARIANT DEHN LEMMA
}

\author{
ALLAN L. EDMONDS
}

\begin{abstract}
An elementary topological proof is given for a completely general version of the Equivariant Dehn Lemma, in the spirit of the original proof of the nonequivariant version due to C. D. Papakyriakopolous in 1957.
\end{abstract}

1. Introduction. The proof by W. H. Meeks III and S.-T. Yau [1981] of the Equivariant Dehn Lemma and the related Equivariant Sphere and Loop Theorems, using techniques from the theory of minimal surfaces, was a major breakthrough for the study of group actions in 3-manifolds. In particular it provided a key ingredient for the proof of the Classical Smith Conjecture (cf. H. Bass and J. W. Morgan [1984]), as well as for other more recent results.

In this paper we present a purely topological proof of the Equivariant Dehn Lemma, using a tower argument in the spirit of Papakyriakopolous [1957]. Using our relatively naive approach we have been unable to prove equivariant versions of the Loop and Sphere Theorems. Recently, however, M. J. Dunwoody [1984] and W. Jaco and $\mathrm{H}$. Rubinstein have announced combinatorial proofs of these results, motivated by minimal surface considerations.

Our purpose in writing this paper is to provide as simple a proof as possible of the main result, in the greatest possible generality, making the theorem more accessible to geometric topologists. It should be emphasized that the minimal surface approach provides a more powerful guiding hand in many problems (only the details are formidable!). The results of M. H. Freedman and Yau [1983] and Meeks and G. P. Scott [1983], for example, still seem inaccessible from the present point of view.

The main result of this paper was announced at the Conference on Combinatorial Methods in.Topology and Algebraic Geometry, Rochester, N. Y., 1982, and a simplified version of the proof for the group $\mathbf{Z}_{p}$ has appeared in the proceedings of that conference (Edmonds [1985]). The reader may find that paper to be a useful introduction to the present version.

The following is the statement we shall prove. Note in particular that there are neither compactness nor orientability assumptions in the statement of the result. All manifolds, subspaces, and mappings are understood to be piecewise linear unless it is explicitly indicated otherwise. The exceptional set for a group action is the set of points with nontrivial isotropy group.

Received by the editors January 3, 1985 and, in revised form, November 12, 1985.

1980 Mathematics Subject Classification. Primary 57M35, 57N10, 57S17.

Research supported by grants from the National Science Foundation. 
THEOREM. Let $G$ be a finite group of homeomorphisms of a 3-manifold $M$. Let $C \subset \partial M$ be a simple closed curve such that (i) $C \simeq 0$ in $M$, (ii) for all $g \in G$ either $g(C)=C$ or $g(C) \cap C=\varnothing$, and (iii) $C$ is transverse to the exceptional set $E$ of the group action. Then there is an embedded 2-disk $D \subset M$ such that (iv) $\partial D=C$, (v) for all $g \in G$ either $g(D)=D$ or $g(D) \cap D=\varnothing$, and (vi) $D$ is transverse to $E$.

Easy examples show that if hypothesis (iii) fails, then no disk satisfying conclusions (iv) and (v) need exist. (For an example, consider the involution on $S^{2} \times I$ with fixed point set $S^{1} \times I$, and $C=S^{1} \times\{0\}$.)

In dimensions at least 5 the analogue of the ordinary Dehn Lemma is trivial, by general position. On the other hand, the obvious analogue of the Equivariant Dehn Lemma in all higher dimensions is easily seen to be false. This fact is surely motivation for thinking that the three-dimensional theorem might be false! For an explicit example take $M=D^{2} \times S^{n}, n \geqslant 2$, with $\mathbf{Z}_{p}$ acting by rotation by $2 \pi / p$ on $D^{2}$ and by the identity on the $S^{n}$ factor. Let $C$ be a perturbation of $S^{1} \times\left\{x_{0}\right\}$ in $S^{1} \times S^{n}$ so that, by general position, $C$ is embedded and $T^{i}(C) \cap C=\varnothing$ for $1 \leqslant i \leqslant p-1$. Intersection numbers show that any disk which $C$ bounds musts intersect the fixed point set $\{0\} \times S^{n}$.

2. Overall strategy of the proof. Suppose that a finite group $G$ acts on a 3-manifold $M$. Let $E(G, M)$ (or just $E(G)$ or even $E$, if no confusion results) denote the exceptional set $\left\{x \in M: G_{x} \neq\{e\}\right\}$. A map $\varphi: D \rightarrow M$ will be called a Dehn disk for the curve $C \subset \partial M$ if $\varphi$ is an immersion, $\varphi \mid \partial D$ parametrizes $C$ homeomorphically, $\varphi$ is transverse to the exceptional set for the $G$ action on $M$ (written $\varphi \pitchfork E(G))$, and $\varphi^{-1}(E(G)) \cap \Sigma(\varphi)=\varnothing$, where $\Sigma(\varphi)=\left\{x \in D: \varphi^{-1} \varphi(x) \neq\{x\}\right\}$ is the singularity set of $\varphi$. A subset $X$ of $M$ will be said to be G-equivariant provided that either $g(X)=X$ or $g(X) \cap X=\varnothing$ for $g \in G$; it is G-invariant if $g(X)=X$ for all $g \in G$. A map $f: M \rightarrow N$ of $G$-spaces is G-equivariant, or a G-map, if $f(g(x))=g(f(x))$ for all $x \in M$ and $g \in G$. Let $G_{C}=\{g \in G: g(C)=C\}$.

PROPOSITION 2.1. Let $G$ be a finite group of orientation preserving homeomorphisms of an orientable 3-manifold $M$, and let $C \subset \partial M$ be a G-equivariant simple closed curve. Suppose that there is a $G_{C}$ equivariant Dehn disk $\varphi: D \rightarrow M$ for $C$ which meets the exceptional set $E(G)$ in at most one point, lying in $E\left(G_{C}\right)$. Then there is a G-equivariant embedded Dehn disk for $C$.

Proof. First consider the case when $G_{C}=\{e\}$, that is $G$ freely permutes the translates of $C$. Then the hypothesis implies that $C$ is nullhomotopic in the $G$-manifold $M_{0}=M-E(G)$. Therefore, in the orbit space $M_{0}^{*}$ the image $C^{*}$ of $C$ is also nullhomotopic. By the ordinary Dehn Lemma, $C^{*}$ bounds an embedded disk $D^{*}$ in $M_{0}^{*}$. The component $D$ of the preimage of $D^{*}$ in $M$ which contains $C$ is the required $G$-equivariant embedded Dehn disk for $C$.

Now consider the case when $G_{C} \neq\{e\}$. The hypotheses imply that $\varphi: D \rightarrow M$ is a $G_{C}$-equivariant map (for a suitable action of $G_{C}$ on $D$ by rotations) which is an immersion and meets $E(G)$ transversely in a single point which is fixed by $G_{C}$. We may suppose that $G_{C}$ fixes the origin 0 in $D$. Excise the interior of a small invariant 
regular neighborhood $N$ of $E(G)$ in $M$ to obtain a manifold $M_{0}$ on which $G$ acts freely. Then $C \subset \partial M_{0}$ is homotopic in $M_{0}$ to a $G$-equivariant simple closed curve $C_{0} \subset \partial M_{0}-\partial M$ which links the fixed point set of $G_{C}$. Passing to $G$ orbit spaces we find that $C^{*}$ and $C_{0}^{*}$ are simple closed curves in $\partial M_{0}^{*}$ and that $C^{*} \simeq C_{0}^{*}$ in $M_{0}^{*}$. Now one may apply the Shapiro-Whitehead [1958] version of the Dehn Lemma for planar domains, or the version of the Dehn Lemma proved in Hempel [1976], to obtain a properly embedded annulus $A^{*} \subset M_{0}^{*}$ with $\partial A^{*}=C^{*} \cup C_{0}^{*}$. Let $A$ be the component of the preimage of $A^{*}$ in $M$ which contains $C$. Filling in a meridian disk for $\partial A-C$ i n $N$ provides the required $G$-equivariant embedded Dehn disk for $C$.

Thus the strategy for the proof of the Equivariant Dehn Lemma in the orientable case is to systematically reduce the intersection of an arbitrary Dehn disk with the exceptional set of the action. This will be accomplished in several steps by lifting pieces of the action to universal coverings in a tower construction similar to that pioneered by Papakyriakopolous. First in $\S 3$ we show that the general case can be reduced to the case of an orientation preserving action with only cyclic isotropy groups. Then in $\S 4$ we describe the process of lifting group actions. Next in $\$ 5$ we describe the general equivariant tower construction. In $\$ 6$ the main result is further reduced to the case of a $G$-invariant simple closed curve (so that $G$ is cyclic) by a tower argument. In $\$ 7$ the case of a cyclic group of prime order is then handled by another tower argument. The case of an arbitrary cyclic group is then dealt with using a tower and inductive argument in $§ 8$.

3. Reduction to the orientable case. In this section we prove first that one may assume that $M$ is connected, second that one may as well assume $M$ is orientable, and third that one may further assume that the action of $G$ on $M$ preserves orientation and has an exceptional set which is a 1-manifold (equivalently, all isotropy groups are cyclic).

LEMMA 3.1. If the Equivariant Dehn Lemma is true for connected manifolds, then it is true in general.

Proof. In the general case let $M^{\prime}$ be the component of $M$ containing $C$ and let $G^{\prime}$ be the maximal subgroup of $G$ leaving $M^{\prime}$ invariant. Any $G^{\prime}$-equivariant Dehn disk for $C$ in $M^{\prime}$ is automatically a $G$-equivariant Dehn disk for $C$ in $M$.

Proposition 3.2. If the Equivariant Dehn Lemma is true for orientable manifolds, then it is true in general.

Proof. Suppose that $G$ acts on the connected, nonorientable manifold $M$ and that $C \subset \partial M$ is a $G$-equivariant simple closed curve which is nullhomotopic in $M$. Let $p: M^{0} \rightarrow M$ be the orientable double covering. Let $C^{0}$ be one of the two loops in $p^{-1}(C)$. Then $C^{0}$ is nullhomotopic in $M^{0}$. Let $G^{0}$ be the group of all lifts of all elements of $G$ to $M^{0}$. Then $C^{0}$ is $G^{0}$-equivariant. Assuming that the Equivariant Dehn Lemma is true for orientable manifolds, there is a $G^{0}$-equivariant embedded Dehn disk $D^{0}$ for $C^{0}$ in $M^{0}$. Then $p\left(D^{0}\right)$ is the required $G$-equivariant embedded Dehn disk for $C$ in $M$. 
Proposition 3.3. If the Equivariant Dehn Lemma is true for orientation preserving actions on orientable manifolds with all isotropy groups cyclic, then it is true for arbitrary actions on orientable manifolds.

Proof. Observe that the set $X$ of points $x$ in $M$ with isotropy group $G_{x}$ containing either (i) a noncyclic orientation preserving subgroup or (ii) an orientation reversing element having $x$ as an isolated fixed point is a discrete $G$-invariant set in the interior of $M$. Then $C$ is nullhomotopic in $M-X$ by general position. Therefore we may as well assume that $X=\varnothing$.

Let $G_{0} \subset G$ be the normal subgroup of orientation preserving elements, which we may assume has index 2 . Then all $G_{0}$ isotropy groups are cyclic, and the exceptional set $E\left(G_{0}\right)$ is a 1 -manifold. The $G$-equivariant loop $C$ may be assumed to bound a $G_{0}$-equivariant embedded Dehn disk $D$.

First consider the case that $D \cap E\left(G_{0}\right)=\varnothing$, so that $G_{0}$ freely permutes the $G_{0}$-translates of $D$. Since $G_{0}$ is normal in $G, E\left(G_{0}\right)$ is $G$-invariant and may (and shall) be assumed to be empty. The force of this assumption is that $E(G)$ is now a (possibly empty) surface whose various components are the fixed point sets for the orientation reversing elements of order 2 in $G$.

Perturb the inclusion $\varphi: D \rightarrow M$ to be transverse to $E(G)$. Then $\varphi^{-1}(E(G))$ consists of a number of pairwise disjoint simple closed curves. We show how to alter the Dehn disk $\varphi$ for $C$ to reduce the number of these simple closed curves (after the first stage of the process, $\varphi$ may no longer be an embedding). Let $\gamma$ be an innermost curve of $\varphi^{-1}(E(G))$ in $D$. Assume $\gamma$ is fixed by $g \in G-\{e\}$. Let $\Delta$ be the 2-disk that $\gamma$ bounds in $D$. Define $\varphi^{\prime}: D \rightarrow M$ to be $\varphi$ outside $\Delta$ and to be $g \circ \varphi$ on $\Delta$. Since $\gamma$ was innermost on $D$, neither $\varphi($ int $\Delta)$ nor $g \circ \varphi($ int $\Delta)$ meet $E(G)$. Now $\operatorname{Im}\left(\varphi^{\prime}\right)$ lies to one side of $E(G)$ near $\varphi^{\prime}(\partial \Delta)$, so a small perturbation $\varphi^{\prime \prime}$ of $\varphi^{\prime}$ has fewer curves of intersection with $E(G)$. Continuing in this way one eventually obtains a Dehn disk for $C$ which misses $E(G)$ completely. Now one may construct the required embedded $G$-equivariant Dehn disk for $C$ by applying the ordinary Dehn Lemma in the orbit space of the free part of the action, as before.

Now consider the case when the $G_{0}$-equivariant embedded Dehn disk $\varphi: D \rightarrow M$ meets $E\left(G_{0}\right)$ in one point. Let $G_{C}=\{g \in G: g(C)=C\}$. There will be two subcases to consider: (i) $G_{C}$ cyclic of order $n$ and (ii) $G_{C}$ dihedral of order $2 n$. In either case we may assume $\varphi$ $\pitchfork(G)$.

In the first subcase $\varphi^{-1}(E(G))$ consists of the isolated point 0 (fixed by $G_{C}$ ) and a collection of simple closed curves. An innermost curve of $\varphi^{-1}(E(G))$ which does not contain 0 may be eliminated just as above. If the only innermost curve of $\varphi^{-1}(E(G))$ contains 0 , the same interchange, followed by a small perturbation, still eliminates a curve of intersection and maintains a single isolated point of intersection with $E\left(G_{0}\right)$. One gets down to the situation where $\varphi^{-1}(E(G))$ is a single isolated point of $E\left(G_{0}\right)$. Thus $C$ is homotopic in the free part of the action to a small linking circle to $E(G)$, which can be assumed to be $G$-equivariant. Now the proof is completed just as in the reduction of the orientable case.

In the last subcase, where $G_{C}$ is dihedral of order $2 n, C$ intersects $E(G)$ transversely in $2 n$ points. Near the point given by $E\left(G_{0}\right) \cap \varphi(D), E(G) \cap \varphi(D)$ is 
the cone on $2 n$ points. In $D-\varphi^{-1}\left(E\left(G_{0}\right)\right)$ the set $\varphi^{-1}(E(G))$ is a 1-manifold. Consideration of the reflections in $G$ along the surfaces in $E(G)$ shows that $\varphi^{-1}(E(G))$ is homeomorphic to a cone on $2 n$ points plus, perhaps, some simple closed curves. One may eliminate an innermost simple closed curve by the interchange procedure above (actually one eliminates $n$ such at once). Thus one may assume that we have a $G_{0}$-immersion $\varphi: D \rightarrow M$ with $\varphi \pitchfork E(G)$ and $\varphi^{-1}(E(G)$ ) the cone on $2 n$ points. One may arrange that $\varphi$ is $G_{C}$-equivariant on a neighborhood of $\varphi^{-1}(E(G))$. Let $N$ be a small invariant regular neighborhood of $E(G) \cup \partial M$ in $M$ whose preimage in $D$ is a regular neighborhood $U$ of $\varphi^{-1}(E(G)) \cup \partial D$ in $D$. Excising the interior of $N$ from $M$ and the interior of $U$ from $D$, one may apply Proposition 2.1 to the $2 n$ freely permuted loops $\partial(D-\operatorname{int} U) \rightarrow \partial(M-\operatorname{int} N)$, to obtain the required $G$-equivariant Dehn disk for $C$ as the union of $\varphi(U)$ and the $2 n$ Dehn disks for $\partial(D-\operatorname{int} U)$.

4. A lifting lemma. In this section we shall describe a way of lifting group actions in universal coverings. This is basically well known (compare Bredon [1972, §1.9], for example), but we need a slightly more general result than appears elsewhere.

LEMMA 4.1. Suppose $G$ is a finite group of homeomorphisms of a 3-manifold $N$ and that $X \subset N$ is a compact connected subspace invariant under $G$ such that the inclusion induced homomorphism $\pi_{1}\left(X, x_{0}\right) \rightarrow \pi_{1}\left(N, x_{0}\right)$ is trivial. Let $p: M \rightarrow N$ be the universal covering and $X^{\prime}$ be a subspace of $M$ which projects homeomorphically to $X$. Then there is a unique action of $G$ on $M$ such that $X^{\prime}$ is G-invariant and $p$ is a G-map.

Remarks. In applications, $X$ will be either a point or a nullhomotopic invariant simple closed curve lying in $\partial N$. The existence of $X^{\prime}$ is of course equivalent to the $\pi_{1}$ hypothesis. Different $\pi_{1}$-trivial invariant sets in $N$ may lead to different lifted actions in $M$. This ambiguity will be important in $\$ 7$.

Proof. Each element $g \in G$ can be lifted to the universal covering $M$ in many ways. Fix a point $x^{\prime} \in X^{\prime}$. Then a lift of $g$ is uniquely determined by the condition that $g\left(x^{\prime}\right)$ lie in $X^{\prime}$. This prescribes an element-by-element lifting of $G$ to $M$. It must be checked that $g(h(x))=g h(x)$ on $M$. It suffices to check this for $x=x^{\prime}$, of course. Equivalently, it must be checked that $g\left(x^{\prime}\right) \in X^{\prime}$ implies $g\left(X^{\prime}\right)=X^{\prime}$. The $\pi_{1}$ hypothesis implies that the various lifts of $X$ are all pairwise disjoint. The conditions $g\left(x^{\prime}\right) \in X^{\prime}$ and $g(X)=X$ imply that $g\left(X^{\prime}\right)$ is a lift of $X$ which meets $X^{\prime}$. Therefore $g\left(X^{\prime}\right)=X^{\prime}$ as required.

5. The tower construction. Let the finite group $G$ act on the 3-manifold $M_{1}$ and suppose that $C \subset \partial M_{1}$ is a $G$-equivariant loop. Let $D \rightarrow M_{1}$ be a Dehn disk for $C$, extended to an equivariant map $\varphi: G \times D \rightarrow M_{1}$. We suppose that $\varphi$ is a simplicial map for triangulations of $G \times D$ and $M_{1}$ with respect to which $G$ acts by simplicial isomorphisms. Let $N_{1}=N\left(\operatorname{Image}(\varphi), M_{1}^{\prime \prime}\right)$ be the $G$-invariant stellar neighborhood obtained by passing to the second barycentric subdivision $M_{1}^{\prime \prime}$ of $M_{1}$ rel Image $(\varphi)$. (That is, each simplex of $M_{1}$ not contained in $\operatorname{Image}(\varphi)$ is starred at its barycenter, 
yielding the first relative barycentric subdivision; the process is then repeated to obtain $M_{1}^{\prime \prime}$. Compare Hempel [1976, p. 48].) Assume that $N_{1}$ is not simply connected, and let $p: M_{2} \rightarrow N_{1}$ be the universal covering of $N_{1}$. Suppose $x_{1} \in$ Image $(\varphi)$ is a fixed point for $G$. Choose a lift $\varphi_{2}:\{e\} \times D \rightarrow M_{2}$ of $\varphi \mid\{e\} \times D$, and choose $x_{2} \in \varphi_{2}(\{e\} \times D) \cap p^{-1}\left(x_{1}\right)$. By Lemma 4.1 there is a unique $G$ action on $M_{2}$ covering the given action on $N_{1}$ fixing the point $x_{2}$. Give $M_{2}$ the triangulation lifted from the triangulation of $N_{1}$. Then $G$ acts simplicially on $M_{2}$. Now extend $\varphi_{2}$ to a $G$-map $\varphi_{2}: G \times D \rightarrow M_{2}$ by equivariance. Then $\varphi_{2}$ is simplicial, where $G \times D$ has the same triangulation it started with. Let $N_{2}$ denote the neighborhood $N\left(\right.$ Image $\left(\varphi_{2}\right), M_{2}^{\prime \prime}$ rel Image $\left.(\varphi)\right)$. The diagram

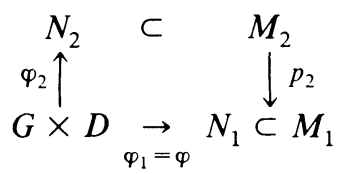

will be called an elementary tower for $\varphi$.

Let $T$ denote the fixed triangulation of $G \times D$. Define the complexity of the simplicial map $\varphi: G \times D \rightarrow M$ to be $\{(\sigma, \tau): \sigma, \tau \in T ; \varphi(\sigma)=\varphi(\tau)\}$. Just as in the standard tower arguments of Papakyriakopolous, the complexity of $\varphi_{2}$ is less than that of $\varphi_{1}$. If $\pi_{1}\left(N_{2}\right) \neq 0$, then one may iterate the construction of an elementary tower:

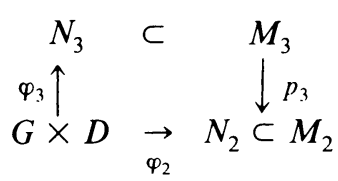

Again the complexity is reduced. If $\pi_{1}\left(N_{3}\right) \neq 0$, one repeats the procedure. Eventually one reaches a stage $\varphi_{k}: G \times D \rightarrow N_{k}$ at which the complexity can no longer be reduced. One concludes that $\pi_{1}\left(N_{k}\right)=0$. The diagram

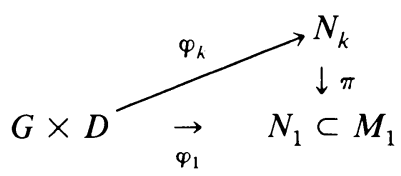

where $\pi$ is a composition of inclusions and covering maps will be called a complete tower for $\varphi$. Note that $\pi$ immerses $N_{k}$ onto a regular neighborhood of Image $(\varphi)$ in $N_{1}$.

Recall from $\$ 4$ that one may lift a group action to the universal covering by specifying that some lift of an invariant $\pi_{1}$-trivial subset be invariant. The tower above was constructed by requiring that a chosen point over a fixed point be fixed. In $\S 8$ we shall use the corresponding tower constructed by specifying that a chosen lift of an invariant, nullhomotopic simple closed curve be invariant.

6. Reduction to the case of an invariant simple closed curve. Here we show that we can systematically reduce the set of points where a Dehn disk for an equivariant simple closed curve meets the exceptional set until it only meets the exceptional set for the subgroup which leaves the simple closed curve invariant. 
Proposition 6.1. Let $G$ be a finite group of homeomorphisms of an orientable 3-manifold $M$ such that the exceptional set $E(G)$ is a nonempty 1-manifold. Let $C \subset \partial M$ be an equivariant simple closed curve which is nullhomotopic in $M$, and let $G_{C}=\{g \in G: g(C)=C\}$. Then there is a Dehn disk $\varphi: D \rightarrow M$ for $C$ such that $\varphi(D) \cap E(G) \subset E\left(G_{C}\right)$.

Proof. We consider Dehn disks $\varphi: D \rightarrow M$ for $C$ and show how to reduce $\varphi(D) \cap\left(E(G)-E\left(G_{C}\right)\right)$, while perhaps increasing the size of $\varphi(D) \cap E\left(G_{C}\right)$. As before we only consider Dehn disks $\varphi$ such that $\varphi \pitchfork E(g)$ and $\varphi^{-1}(E(G)) \cap \Sigma(\varphi)$ $=\varnothing$. Suppose there is a point $x_{1} \in \varphi(D) \cap\left(E(G)-E\left(G_{C}\right)\right)$, and let $g$ be a nontrivial element of $G$ such that $g\left(x_{1}\right)=x_{1}$. Since $x_{1}$ is not in $E\left(G_{C}\right), x_{1}$ is not fixed by any nontrivial element of $G_{C}$.

Let $\langle g\rangle$ denote the cyclic subgroup of $G$ generated by $g$, and consider the following tower in which $g$ fixes the preimage of $x_{1}$ :

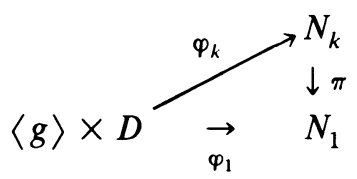

Let $E_{1}=E \cap N_{1}$, and note that $g$ permutes the arcs in $E_{1}$. It is understood that $N_{k}$ is simply connected, and so $\partial N_{k}$ consists of 2-spheres permuted by the action of $g$. Further $\pi^{-1}\left(E_{1}\right)$ consists of arcs, each mapped homeomorphically into $E_{1}$ by $\pi$. The main point to see here is that if $A$ is an arc of $E_{1}$, then $\langle g\rangle$ acts transitively on the set $\pi^{-1}\left(\cup_{j} g^{j}(A)\right)$. (This uses the condition that $\varphi^{-1}(E) \cap \Sigma(\varphi)=\varnothing$.)

Let $C_{k}=\varphi_{k}(\{e\} \times \partial D)$. Since $\partial N_{k}$ is a union of 2-spheres and $g\left(C_{k}\right) \cap C_{k}=\varnothing$, there is at least one 2-disk $\Delta \subset \partial N_{k}$ such that $g(\Delta) \cap \Delta=\varnothing$. Define $\varphi^{\prime}$ to be the Dehn disk given by $\pi \mid \Delta$. We shall see that (i) $\varphi^{\prime} \pitchfork E$, (ii) $\varphi^{\prime-1}\left(E(G)-E\left(G_{C}\right)\right) \cap$ $\Sigma\left(\varphi^{\prime}\right)=\varnothing$, and (iii) $\# \varphi^{\prime-1}\left(E(G)-E\left(G_{C}\right)\right)<\# \varphi^{-1}\left(E(G)-E\left(G_{C}\right)\right)$. Given these three assertions, one can perturb $\varphi^{\prime}$ slightly near points of $E\left(G_{C}\right)$ to give a map $\varphi^{\prime \prime}$ : $D \rightarrow M$ such that

$$
\varphi^{\prime \prime} \pitchfork E(G), \quad \varphi^{\prime \prime-1}(E(G)) \cap \Sigma\left(\varphi^{\prime \prime}\right)=\varnothing,
$$

and

$$
\# \varphi^{\prime \prime-1}\left(E(G)-E\left(G_{C}\right)\right)<\# \varphi^{-1}\left(E(G)-E\left(G_{C}\right)\right) .
$$

Repeating the procedure then produces the required map.

Now (i) is clear since $\Delta \cap \pi^{-1}\left(E_{1}\right)$.

Assertion (ii) follows from the observation that if $A$ is an $\operatorname{arc}$ of $E_{1}$ and $\pi^{-1}(A)$ meets $\Delta$ more than once, then $A \subset E(G)$. If a single arc of $\pi^{-1}(A)$ has both end points in $\Delta$, then one can join the end points to create a 1-cycle which meets $\varphi_{k}(D)$ exactly once, contradicting the fact that $H_{1}\left(N_{k}\right)=0$. If two $\operatorname{arcs}$ of $\pi^{-1}(A)$ meet $\Delta$, then some nontrivial power $g^{r}$ maps one to the other, hence leaves $\Delta$ invariant; then $g^{r}$ fixes $A$ and leaves $C$ invariant.

To check (iii), first note that for each arc $A$ of $E_{1}, \pi^{-1}(A)$ meets $\varphi(D)$ exactly once. This follows from the fact that $\varphi^{-1}(E(G)) \cap \Sigma(\varphi)=\varnothing$. As noted in the preceding paragraph, $\pi^{-1}(A)$ meets $\Delta$ at most once if $A \subset E(G)-E\left(G_{C}\right)$. But by 
construction there is at least one arc $A$ (the one containing the point $x_{1}$ ) which lies in $E(G)-E\left(G_{C}\right)$ and whose preimage does not meet $\Delta$, since $\pi^{-1}(A)$ is a single arc fixed by $g$. Statement (iii) follows.

Remark. Given the result of Proposition 6.1, one can excise the $G_{C}$-invariant set $E(G)-E\left(G_{C}\right)$ from $M$ to obtain $M^{\prime}$, say. The curve $C$ is now nullhomotopic in $M^{\prime}$. If one can find an embedded, $G_{C}$-equivariant Dehn disk for $C$ in $M^{\prime}$ (hence in $M$ ), then Proposition 2.1 applies to yield a $G$-equivariant embedded Dehn disk for $C$ in $M$. In other words we have justified the following reduction in the proof of the Equivariant Dehn Lemma:

COROllary 6.2. In order to prove the Equivariant Dehn Lemma in general, it is sufficient to prove it in the case of a compact, connected, orientable manifold $M$ on which the finite group $G$ acts by orientation preserving homeomorphisms, leaving the simple closed curve $C \subset \partial M$ invariant.

Since $C$ may be assumed to be invariant, $C \pitchfork E(G)$, and $G$ orientation preserving, it follows that $G$ is a cyclic group $\mathbf{Z}_{m}$ of order $m$. We next address the case when $G$ is cyclic of prime order $p$.

7. The case of an invariant curve and a group of prime order. In this section we prove the following special case of the Equivariant Dehn Lemma.

Proposition 7.1. Let $G$ be a finite cyclic group of prime order p acting nontrivially by orientation preserving homeomorphisms on the orientable 3-manifold M. Suppose that $C \subset \partial M$ is a simple closed curve which is invariant under $G$, misses the exceptional set $E(G)$, and is nullhomotopic in $M$. Then there exists an embedded disk $D$ in $M$ with $\partial D=C$ which is invariant under $G$.

Proof. By Proposition 2.1 it suffices to find a Dehn disk $\varphi: D \rightarrow M$ for $C$ such that $\varphi \pitchfork E(G)$, and $\varphi^{-1}(E(G))$ consists of a single point. We will thus in general show that one can reduce the size of $\varphi^{-1}(E(G))$ when it consists of more than one point. (Clearly $\varphi^{-1}(E(G)) \neq \varnothing$, since, otherwise, covering space theory would imply that $G$ moves $C$ off itself.)

We consider Dehn disks $\varphi: D \rightarrow M$ for $C$ which are immersions transverse to $E(G)$ such that in addition $\varphi^{-1}(E(G)) \cap \Sigma(\varphi)=\varnothing$, where

$$
\Sigma(\varphi)=\left\{x \in D: \varphi^{-1} \varphi(x) \neq\{x\}\right\}
$$

is the singularity set of $\varphi$. Such Dehn disks exist since one can apply the ordinary Dehn Lemma to choose $\varphi$ to be an embedding and then perturb it to be transverse to $E(G)$.

Let such a Dehn disk $\varphi: D \rightarrow M$ be given. We show that either we can produce the desired Dehn disk, or at least we can find another one which satisfies the given technical conditions for which the preimage of $E(G)$ is smaller.

Begin by extending $\varphi$ to a $G$-map $\varphi_{1}: G \times D \rightarrow M$. Triangulate $G \times D$ and $M$ so that $\varphi_{1}$ is simplicial. Let $M_{1}$ denote $M$ with this triangulation. Let $M_{1}^{\prime \prime}$ denote the second barycentric subdivision of $M_{1}$ relative to $\operatorname{Image}(\varphi)$, and let

$$
N_{1}=N\left(\operatorname{Image}(\varphi), M_{1}^{\prime \prime}\right)
$$


be the simplicial neighborhood of Image $(\varphi)$ in $M_{1}^{\prime \prime}$. Note that $E(G) \cap N_{1}$ consists of arcs $A_{j}$ transverse to each $\varphi(g \times D)$.

Suppose that by chance $\pi_{1}\left(N_{1}\right)=0$. Then $C$ is a $G$-invariant curve lying on a 2-sphere $S \subset \partial N_{1}$. Let $\Delta$ be the closure of one of the complementary domains of $C$ in $S$. Then $\Delta$ is the required Dehn disk for $C$ meeting $E(G)$ exactly once.

Now suppose that $\pi_{1}\left(N_{1}\right) \neq 0$. We will construct a tower as described in $§ 5$. In this case some care will be required with the choice of lifting of the group action to the covering spaces.

Let $\pi: M_{2} \rightarrow N_{1}$ be the universal covering of $N_{1}$ and let $\varphi_{2}: D \rightarrow M_{2}$ be a lift of $\varphi_{1}$.

LEMMA 7.2. Either there is a lifting of the $G$ action to $M_{2}$ such that $g\left(\varphi_{2}(\partial D)\right) \cap$ $\varphi_{2}(\partial D)=\varnothing$ for all nontrivial $g \in G$, or there is a lifting of the $G$ action to $M_{2}$ such that $G\left(\varphi_{2}(\partial D)\right)=\varphi_{2}(\partial D)$ and $\pi^{-1}(E(G)) \cap \varphi_{2}(D)$ consists entirely of fixed points.

Proof. Let $E(G) \cap \varphi(D)=\left\{x_{1}, \ldots, x_{n}\right\}$, and let $\pi^{-1}(E(G)) \cap \varphi_{2}(D)=$ $\left\{y_{1}, \ldots, y_{n}\right\}$, where $\pi\left(y_{i}\right)=x_{i}$. Consider the various actions of $G$ on $M_{2}$ lifting the given action on $N_{1}$ given by deciding that $y_{i}$ should be fixed, $i=1, \ldots, n$. Suppose that all of these actions leave $\varphi(\partial D)$ invariant. We shall then show that all these actions coincide and hence fix the entire set $\left\{y_{1}, \ldots, y_{n}\right\}$.

For each $i, \varphi^{-1}\left(x_{i}\right)$ is a single point, say $z_{i}$, since $\varphi^{-1}(E(G)) \cap \Sigma(\varphi)=\varnothing$. Choose a base point $z_{0} \in \partial D$ and choose paths $\lambda_{i}:(I, 0,1) \rightarrow\left(D, z_{0}, z_{i}\right)$. Now $\left[g \varphi \lambda_{i}\right]=\left[\varphi \lambda_{i}\right]$ in the set $\left[I, 0,1 ; N_{1}, C, z_{i}\right]$ of homotopy classes of paths from $C$ to $z_{i}$, since we have assumed that the lifting of the $G$ action to $M_{2}$ which fixes $y_{2}$ leaves $\varphi_{2}(C)$ invariant.

Now let $\mu_{i}$ be a path in $D$ running from $z_{1}$ to $z_{i}$. We claim that $g \varphi \mu_{i} \simeq \varphi \mu_{i}$ rel $\{0,1\}$ :

$$
\begin{aligned}
g \varphi \mu_{i} & \simeq g\left(\varphi \lambda_{1}^{-1} * \varphi \lambda_{i}\right) \quad\left(\text { since } \mu_{i} \simeq \lambda_{1}^{-1} * \lambda_{i}\right) \\
& \simeq g \varphi \lambda_{1}^{-1} * g \varphi \lambda_{i} \\
& \simeq \varphi \lambda_{1}^{-1} * \tau * \varphi \lambda_{i} \quad(\text { for some loop } \tau \text { in } C) \\
& \simeq \varphi \lambda_{1}^{-1} * \varphi \lambda_{i} \quad\left(\text { since } C \simeq 0 \text { in } N_{1}\right) \\
& \simeq \varphi \mu_{i} .
\end{aligned}
$$

Covering space theory now implies that the action fixing $y_{1}$ also fixes each $y_{i}$.

Now erect a complete tower as in $\S 5$ (where $N_{k}$ is simply connected):

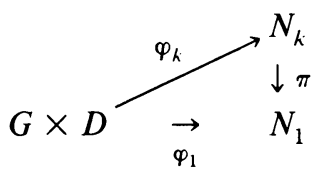

It is understood that if at any stage it is possible to choose the elementary tower so that the lifted curve over $C$ is no longer invariant, then this is done. At the $i$ th stage, let $E_{i}$ denote the (nonempty by construction) exceptional set for the group action on $N_{i}$. Either $\varphi_{k}(\partial D)$ is $G$-invariant and $E_{k}=\pi^{-1}\left(E_{1}\right)$ or there is some smallest $i_{0}<k$ 
such that $\varphi_{i}(\partial D)$ is not invariant for $i>i_{0}$. In the latter case $G$ freely permutes the preimages of $C$ in $N_{k}$, since $G$ has prime order. We consider these two cases separately.

Case (i). $\varphi_{k}(\partial D)$ is $G$-invariant. By construction $\pi^{-1}\left(E_{1}\right)=E_{k}$. In particular, for each arc $A$ of $E_{1}, \pi^{-1}(A)$ is a single arc, fixed by $G$. Since $\pi_{1}\left(N_{k}\right)=0, \partial N_{k}$ is a union of 2-spheres. Therefore $\varphi_{k}(\partial D)$ is an invariant curve on an invariant 2-sphere $S$ on which $G$ must be acting by rotations. Let $\Delta$ be the closure of one of the complementary domains of $\varphi_{k}(\partial D)$ in $S$. Then $\Delta \cap E_{k}=\Delta \cap \pi^{-1}\left(E_{1}\right)$ is a single point and the restricted projection map $\pi \mid: \Delta \rightarrow M$ yields an immersed Dehn disk for $C$ which meets $E(G)$ transversely in a single point. Proposition 2.1 shows that there is then a $G$-invariant embedded Dehn disk for $C$, as required.

Case (ii). $\varphi_{k}(\partial D)$ is mapped off of itself by the nontrivial elements of $G$. Since $\pi_{1}\left(N_{k}\right)=0, \partial N_{k}$ is a union of 2-spheres. Say that $\varphi_{k}(\partial D)$ lies in the 2-sphere $S \subset \partial N_{k}$. At least one of the two complementary domains of $\varphi_{k}(\partial D)$ in $S$ must miss $E_{k}$, since $G$ maps $\varphi_{k}(\partial D)$ off itself. Let $\Delta$ be the closure of such a complementary domain. For each arc $A$ of $E_{1}, \pi^{-1}(A)$ consists either of a single fixed arc or of $p$ arcs freely permuted by $G$. As in the proof of Proposition 6.1, $\Delta$ can meet $\pi^{-1}(A)$ in at most one point. By construction there is at least one arc $A_{0}$ of $E_{1}$ such that $\pi^{-1}\left(A_{0}\right)$ is a single fixed arc. Clearly $\Delta \cap \pi^{-1}\left(A_{0}\right)=\varnothing$. Now the restricted projection $\pi \mid: \Delta \rightarrow M$ yields a Dehn disk for $C$ such that $\pi \mid$ is an immersion transverse to $E(G),(\pi \mid)^{-1}(E(G)) \cap \Sigma(\pi \mid)=\varnothing$, and $\pi \mid$ meets $E(G)$ fewer times than the original $\varphi$ did.

8. The case of an arbitrary invariant curve. In this section we inductively extend the argument of $\S 7$ to complete the proof of the Equivariant Dehn Lemma.

LEMMA 8.1. Let $G$ be a finite cyclic group of orientation preserving homeomorphisms of an orientable 3-manifold $M$, and suppose that $C \subset \partial M$ is a G-invariant simple closed curve which is nullhomotopic in $M$ and misses $E(G)$. Then any Dehn disk for $C$ meets the fixed point set $M^{G}$.

Proof. Let $\varphi: D \rightarrow M$ be a Dehn disk for $C$ and extend by equivariance to a $G$-map $\varphi_{1}: G \times D \rightarrow M$. Construct a complete tower as in $\S 5$, except that at each stage one lifts the action of $G$ by requiring that a lift of $C$ to the next stage be $G$-invariant (cf. the remarks in $\S 4$ about the Lifting Lemma). The result is a complete tower

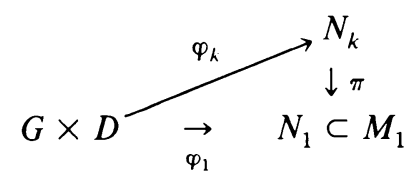

where as usual $N_{1}$ denotes a regular neighborhood of $\varphi_{1}(G \times D)$ and $N_{k}$ is simply connected. Let $C_{k} \subset N_{k}$ be $\varphi_{k}(\{e\} \times \partial D)$. Then $C_{k}$ is a $G$-invariant simple closed curve lying on a $G$-invariant 2-sphere $S$ in $\partial N_{k}$. Then $S$ necessarily contains a fixed point for $G$. It follows that $N_{1}$ contains a fixed point, and hence that $\varphi_{1}(G \times D)$ and the original $\varphi(D)$ do also. 
Proposition 8.2. Let $G$ be a finite cyclic group of order $m$ acting nontrivially by orientation preserving homeomorphisms on the orientable 3-manifold M. Suppose that $C \subset \partial M$ is a simple closed curve that is invariant under $G$, misses the exceptional set $E(G)$, and is nullhomotopic in $M$. Then there exists an embedded disk $D$ in $M$ with $\partial D=C$ that is invariant under $G$.

Proof. We shall proceed by induction on the order of $G$. The induction is started by Proposition 7.1, which was the case for prime order groups. Suppose now that $m$ is not prime, but is divisible by a prime $p$. Let $H$ be a subgroup of $G$ of index $p$. By induction there is an $H$-invariant embedded Dehn disk $D$ for $C$. Then $D \cap E(H)$ is a single point $x_{0}$ fixed by $H$. On the other hand Lemma 8.1 shows that $D$ contains a fixed point of all of $G$. We conclude that the point $x_{0}$ is fixed by $G$. The other points of $D \cap E(G)$ are freely permuted by $H$ and therefore fixed by elements of order $p$ not in $H$. We conclude further that $H$ is a split subgroup, with complement $Q$ of order $p$. Let $N$ be a small $G$-invariant simplicial neighborhood of $G(D)$ in the second barycentric subdivision of a triangulation with respect to which $G$ acts simplicially. Then $E(H) \cap N$ is a single arc of points fixed by the whole group $G$. Apply Proposition 7.1 to find a $Q$-invariant embedded Dehn disk $\Delta$ for $C$ lying in the manifold $N$. Then, although $\Delta$ may not be invariant under all of $G$, we see that at least $\Delta \cap E(G)$ is a single point.

Now the tower argument of Lemma 8.1 produces a $G$-invariant Dehn disk for $C$ which meets $E(G)$ in exactly one point. Proposition 2.1 then applies to complete the proof.

\section{REFERENCES}

H. Bass and J. W. Morgan (eds.), The Smith conjecture, Academic Press, New York, 1984.

G. E. Bredon, Introduction to compact transformation groups, Academic Press, New York, 1972.

M. J. Dunwoody, An equivariant sphere theorem, preprint, 1984.

A. L. Edmonds, On the equivariant Dehn lemma, Proc. Conf. on Combinatorial Methods in Topology and Algebraic Geometry (Rochester, N.Y., 1982), Contemp. Math., Vol. 44, Amer. Math. Soc., Providence, R. I., 1985, pp. 141-147.

M. H. Freedman and S.-T. Yau, Homotopically trivial symmetries of Haken manifolds are toral, Topology 22 (1983), 179-189.

J. Hempel, 3-manifolds, Ann. of Math. Studies, no. 86, Princeton Univ. Press, Princeton, N. J., 1976.

W. H. Meeks III and G. P. Scott, Finite groups actions on 3-manifolds, preprint, 1983.

W. H. Meeks III and S.-T. Yau, The equivariant Dehn's lemma and the loop theorem, Comment. Math. Helv. 56 (1981), 225-239.

C. D. Papakyriakopolous, On Dehn's lemma and the asphericity of knots, Ann. of Math. 66 (1957), $1-26$.

G. P. Scott, There are no fake Seifert fibre spaces with infinite $\pi_{1}$, Ann. of Math. 117 (1983), 35-70.

A. Shapiro and J. H. C. Whitehead, A proof and extension of Dehn's lemma, Bull. Amer. Math. Soc. 64 (1958), 174-178.

DePartment of Mathematics, Indiana University, BloOMINGTON, INDIANA 47405 